\title{
Prevalence and Pattern of Coronary Artery Disease in Diabetic Patients with Cardiomyopathy
}

\author{
Haitham Mohamed Ramadan Tofaha, Mohamed Salah Elden Abd Salam, Ahmed EL Tayeb \\ Department of Cardiology, Faculty of Medicine, Al-Azhar University, Assuit, Egypt \\ *Corresponding author: Haitham Mohamed Ramadan Tofaha, Mobile: (+20) 01022963773,
}

E-Mail: drhaithamh6@gmail.com

\begin{abstract}
Background: Coronary artery disease $(\mathrm{CAD})$ is a group of diseases that includes stable angina, unstable angina, myocardial infarction and sudden cardiac death. It's the most commonly occurring type of cardiovascular diseases. Risk factors for CAD include hypertension, diabetes mellitus, smoking, alcohol ingestion, obesity, dyslipidemia, sedentary life and family history. The underlying mechanism involves atherosclerosis of the arteries of the heart

Objectives: The aim of the work was to assess the prevalence and pattern of coronary artery disease through invasive coronary angiography in diabetic patients with cardiomyopathy.

Patients and Methods: Fifty diabetic patient with echocardiographic evidence of cardiomyopathy were enrolled in this study, which conducted at Cardiology Department Azhar Assuit University Hospital during the period from November 2018 to March 2021. Informed consent was obtained from every patient after explanation of the procedure. Medical research and Ethics Committee approved the study.

Results: As regard HbA1c there was highly statistically significant ( $\mathrm{p}$-value $<0.001)$ positive correlation $(\mathrm{r}=0.82)$ between HbA1c and duration of DM. Highly statistically significant (p-value $<0.001$ ) positive correlation between (HbA1c and SYNTAX score) and (duration and SYNTAX score).
\end{abstract}

Conclusion: There was a correlation between HbA1c and SYNTAX score and duration of diabetes.

Keywords: Diabetes mellitus, coronary artery disease, cardiomyopathy, SYNTAX score.

\section{INTRODUCTION}

The term diabetes mellitus describes a metabolic disorder of multiple etiologies characterized by chronic hyperglycemia with disturbances of carbohydrate, fat and protein metabolism resulting from defects in insulin secretion, insulin action, or both. The long-term effects of diabetes mellitus include progressive development of retinopathy, nephropathy, and/or neuropathy, and features of autonomic dysfunction. People with diabetes are at increased risk of cardiovascular, peripheral vascular and cerebrovascular disease ${ }^{(\mathbf{1})}$.

A number of experimental, pathologic, and epidemiologic studies support the existence of diabetic cardiomyopathy ${ }^{(2)}$, the clinical diagnosis of which is made when systolic and diastolic left ventricular (LV) dysfunction are present in diabetic patients without other known cardiac disease ${ }^{(3)}$.

As treatment to reverse this disorder is more likely to be effective at an early (preclinical) stage, defining the mechanism of diabetic cardiomyopathy may be important to its selective treatment. However, the etiologic agent for diabetic heart disease remains undefined, the most likely contributors being disturbances of cardiac muscle, pathology involving the cardiac stroma (e.g. fibrosis), or disease of the small vessels ${ }^{(4)}$.

Diabetes up regulates the rennin-angiotensin system, which may contribute to the development of a dilated cardiomyopathy, whereas locally, angiotensin II may lead to oxidative damage, activating cardiac cell death. These advances in the basic understanding of cellular mechanisms underlying diabetic cardiomyopathy might be relevant to therapy. Tight glycemic control could be a strategy to prevent cardiomyopathy, along with other pharmacologic treatment, for instance, angiotensin-converting enzyme inhibitors, selective blockers of angiotensin II type 1 receptors, or aldosterone antagonists at low nondiuretic doses ${ }^{(5)}$.

Atherosclerosis is a syndrome affecting arterial blood vessels; due to a chronic inflammatory response in the walls of the arteries. This response is promoted by low density lipoproteins and inhibited by high density lipoproteins. Atherosclerosis is a chronic disease that may remain asymptomatic for decades. Atherosclerosis affects mostly large vessels; such as coronary, carotid, cerebral, femoral and renal arteries. Hardening and narrowing of the arteries result from athermanous plaques ${ }^{(6)}$.

Coronary artery disease (CAD) is a group of diseases that includes: stable angina, unstable angina, myocardial infarction and sudden cardiac death. It's the most commonly occurring type of cardiovascular diseases. Risk factors for CAD include: hypertension, diabetes mellitus, smoking, alcohol ingestion, obesity, dyslipidemia, sedentary life and family history. The underlying mechanism involves atherosclerosis of the arteries of the heart ${ }^{(7)}$.

The aim of the work was to assess the prevalence and pattern of coronary artery disease in diabetic

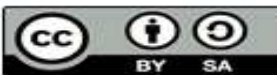

This article is an open access article distributed under the terms and conditions of the Creative Commons Attribution (CC BY-SA) license (http://creativecommons.org/licenses/by/4.0/) 
patients with cardiomyopathy undergoing coronary angiography.

\section{PATIENTS AND METHODS}

Fifty diabetic patient with echocardiographic evidence of cardiomyopathy were enrolled in this study, which was conducted at Cardiology Department Azhar Assuit University Hospital during period from November 2018 to March 2021.

Inclusion Criteria: Diabetic patients with cardiomyopathy who were eligible for elective coronary angiography for evaluation of suspected CAD.

Exclusion Criteria: Hemodynamically unstable patients, patients with acute coronary syndrome, patient with significant primary valvular lesions, valvular cardiomyopathy and congenital heart disease, previous revascularization procedure (PCI or $\mathrm{CABG}$ ), and patient with contraindication to do coronary angiography such as severe renal or hepatic impairment.

\section{All patients in this study were subjected to:}

1- Full history: including age, sex, risk factors for CAD (smoking, hypertension, PAD, FH of IHD, type of DM, cardiac history, medications, peripheral artery disease and family history.

2- Full clinical examination: with special emphasis on the following data: Pulse: rate and rhythm, blood pressure, head and neck examination, upper and lower limb examination, and chest and heart examination.

3- Laboratory investigations: including kidney function tests, complete blood count, INR, blood glucose level (fasting blood glucose, HbA1c, random blood glucose).

4- Twelve-lead electrocardiogram (ECG) (Biocare 3000, China)

5- Transthoracic echocardiography (TTE): $\rightarrow$ (GE, vivid S5, Norway)

TTE was done for every patient in the study for evaluation of left ventricular systolic and diastolic function. Patients were included if ejection fraction (EF) $<50 \%$ assessed by the following methods: MMode and Simpson method ${ }^{(8)}$.

6- Coronary angiography: Pre catheter assessment: Full history, physical examination and pre catheter lab including (Serum creatinine, PT, PC, INR).

Patient preparation: Coronary angiography was performed under local anesthesia. The procedure was sterile, and all potential access sites were disinfected, shaved, and sterilized. At the beginning of the procedure, the patient layed down in supine position on the cardio angiograph table, and was prepared for the procedure in sterile conditions.

Premedication: Sedation, Anti allergic, Antiemetic.

Contrast media: Xenitax $350 \mathrm{mg} / 50 \mathrm{ml}$ and maximum amount $=5$ (body weight) $/$ Serum creatinine. Catheters: XP Judkins (J) and Amplatz (A), JL 3.5 and JL 4, JR 3.5 and JR 4, and AL I, II, III AR I, II.
Approaches: Percutaneous techniques usually from femoral and radial artery was used.

Projections: Selective left and right coronary angiograms were performed in multiple angulated views: AP (anteroposterior) cranial and caudal, LAO (Left Anterior Oblique) cranial and caudal, RAO cranial and caudal, and lateral view.

SYNTAX score is a scoring system used to estimate the severity of CAD through coronary angiography, on the basis of coronary anatomic risk factors. These factors include: the number of lesions, total occlusion, bifurcation, trifurcation, ostial stenosis, tortuosity, calcification, thrombus, diffuse lesion and small vessel/diffuse disease. SYNTAX score was calculated using SYNTAX score algorithm by highly experienced interventional cardiologists.

\section{Ethical considerations:}

An approval of the study was obtained from AlAzhar University (Assuit) Academic and Ethical Committee. Every patient signed an informed written consent for acceptance of the operation after explanation of the procedure.

\section{Statistical methods}

Data were collected and analyzed using SPSS (Statistical Package for the Social Sciences, version 20, IBM, and Armonk, New York). Quantitative data were expressed in the form of mean \pm SD. Nominal data were expressed in the form of frequency (Percentage). $\mathrm{P}<0.05$ was considered significant and very high significant result was considered if $\mathrm{P}<$ 0.001. Pearson Correlation/ Spearman correlation (according to data distribution) was used.

\section{RESULTS}

This table shows the description of demographic data in all studied patients.

Table (1): Description of demographic and clinical data in all studied patients

\begin{tabular}{|c|c|c|c|}
\hline \multicolumn{2}{|c|}{} & $\begin{array}{c}\text { Studied patients } \\
(\mathbf{N}=\mathbf{5 0})\end{array}$ \\
\hline \multirow{2}{*}{ Age (years) } & Mean \pm SD & $57.4 \pm 9.04$ \\
\cline { 2 - 4 } & Min - Max & \multicolumn{2}{|c|}{$40-77$} \\
\hline \multirow{2}{*}{ Sex } & Male & 28 & $56 \%$ \\
\cline { 2 - 4 } & Female & 22 & $44 \%$ \\
\hline \multirow{2}{*}{ HTN } & Non-HTN & 15 & $30 \%$ \\
\cline { 2 - 4 } & HTN & 35 & $70 \%$ \\
\hline \multirow{2}{*}{ CKD } & Non-CKD & 48 & $96 \%$ \\
\cline { 2 - 4 } & CKD & 2 & $4 \%$ \\
\hline \multirow{2}{*}{ Famoking } & Non-smoker & 28 & $56 \%$ \\
\cline { 2 - 4 } & Smoker & 22 & $44 \%$ \\
\hline BMI (kg/m ${ }^{2}$ ) & Negative & 29 & $58 \%$ \\
\cline { 2 - 4 } & Positive & 21 & $42 \%$ \\
\cline { 2 - 4 } & Mean \pm SD & $25.7 \pm 2.2$ \\
\hline
\end{tabular}

Table (2) shows the description of clinical data in all studied patients. 
Table (2): Clinical data in all studied patients

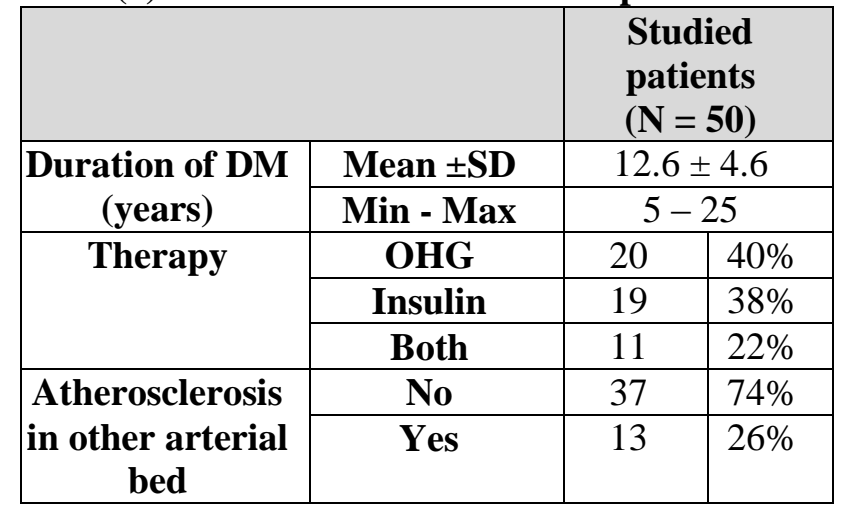

Table (3) shows the description of $\mathrm{HbAlc}$ in all studied patients.

Table (3) Description of HbA1c in all studied patients

\begin{tabular}{|c|l|c|}
\hline \multicolumn{2}{|c|}{} & $\begin{array}{c}\text { Studied patients } \\
(\mathbf{N}=\mathbf{5 0})\end{array}$ \\
\hline HbA1c (\%) & Mean \pm SD & $7.4 \pm 1.03$ \\
\cline { 2 - 3 } & Min - Max & $5.5-10$ \\
\hline
\end{tabular}

This table shows the description of SYNTAX score and number of vessels in all studied patients (Table 4).

Table (4): Description of SYNTAX score and number of vessels in all studied patients

\begin{tabular}{|c|l|c|c|}
\hline \multicolumn{2}{|c|}{} & \multicolumn{2}{|c|}{$\begin{array}{c}\text { Studied } \\
\text { patients } \\
\mathbf{( N = 5 0 )}\end{array}$} \\
\hline $\begin{array}{c}\text { SYNTAX } \\
\text { score }\end{array}$ & \multicolumn{1}{|c|}{ Mean \pm Min - Max } & \multicolumn{2}{|c|}{$14.2 \pm 5.6$} \\
\cline { 2 - 4 } & \multicolumn{1}{|c|}{$0-28$} \\
\cline { 2 - 4 } & Normal coronary angio & 1 & $2 \%$ \\
\cline { 2 - 4 } & $\begin{array}{l}\text { non-significant } \\
\text { atherosclerotic CAD }\end{array}$ & 20 & $40 \%$ \\
\cline { 2 - 4 } & $\begin{array}{l}\text { Significant } \\
\text { atherosclerotic CAD }\end{array}$ & & \\
\cline { 2 - 4 } & LMCAD & 4 & $8 \%$ \\
\hline & Single vessel CAD & 16 & $32 \%$ \\
\hline & Two vessels disease & 8 & $16 \%$ \\
\hline & Multivessel disease & 5 & $10 \%$ \\
\hline
\end{tabular}

Table 5 and figures 1 and 2 show highly statistically significant positive correlation between (HbAlc and SYNTAX score) and (duration and SYNTAX score).

Table (5): Correlation study between SYNTAX score and both of HbA1c and Duration of DM

\begin{tabular}{|c|c|c|c|c|}
\hline Variables & \multicolumn{2}{|c|}{ HbA1c } & \multicolumn{2}{c|}{ Duration } \\
\cline { 2 - 5 } & $\mathrm{r}$ & $\mathrm{p}$-value & $\mathrm{r}$ & $\mathrm{p}$-value \\
\hline $\begin{array}{c}\text { SYNTAX } \\
\text { score }\end{array}$ & 0.95 & $<0.001$ & 0.85 & $<0.001$ \\
HS & & HS \\
\hline
\end{tabular}

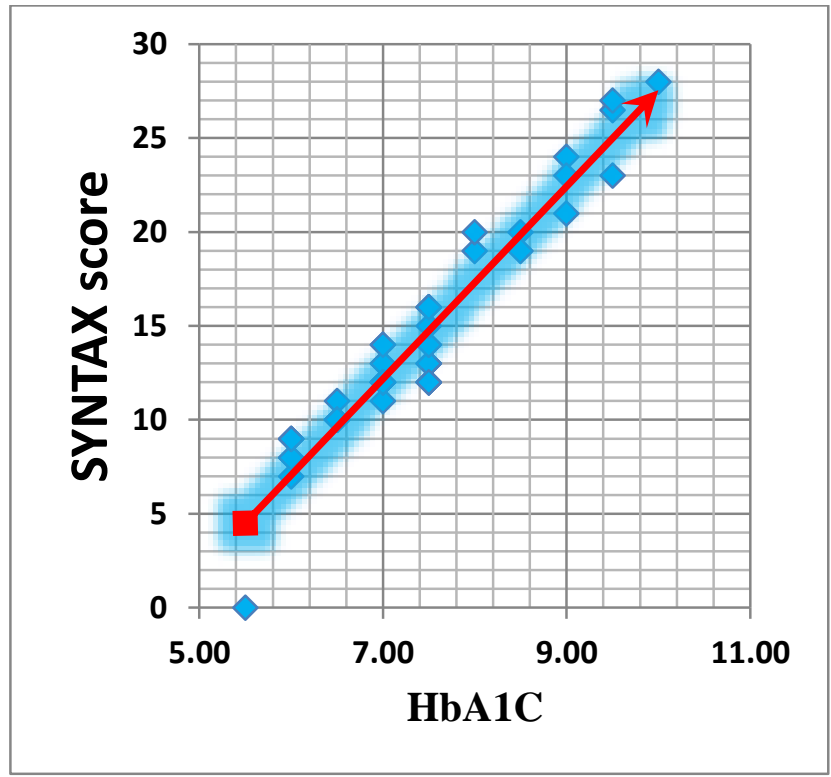

Figure (1): Positive correlation between HbA1c and SYNTAX score.

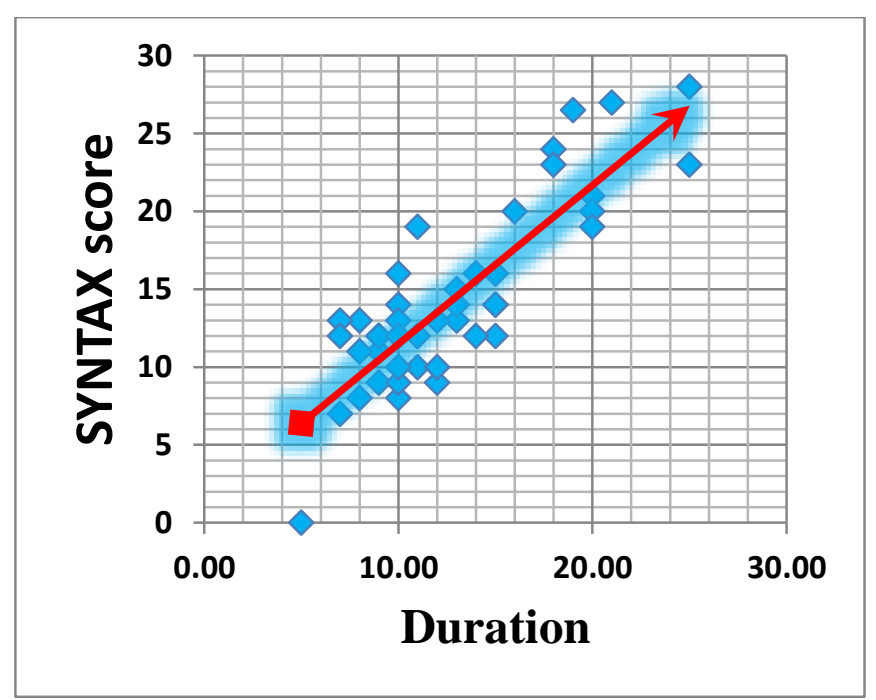

Figure (2): Positive correlation between duration and SYNTAX score

\section{DISCUSSION}

Coronary artery disease (CAD) is the leading cause of mortality and morbidity in patients with diabetes. Approximately one-half of deaths are attributed to CAD in diabetic patients, whose risk of myocardial infarction or cardiac death is two-to fourfold as great compared with nondiabetic patients ${ }^{(9)}$. The higher incidence and increased risk of developing CAD have been positively linked with diabetes mellitus (DM) in a number of studies ${ }^{(\mathbf{1 0})}$.

Diabetes mellitus (DM) is a group of metabolic diseases characterized by hyperglycemia resulting from defects in insulin secretion, response to insulin, or both (American Diabetes Association, 2014) ${ }^{(\mathbf{1})}$. The prevalence of microvascular complications increases with the duration of the diabetes ${ }^{(11)}$.

Glycated hemoglobin (HbAlc) is widely acknowledged as a pivotal and more accurate criterion 
for pre-empting the severity of DM over fasting glucose levels ${ }^{(12)}$. HbA1c levels offer more advantage by providing a stable index of long-term glycemic status, which is related to two to three months of average glucose concentration in plasma ${ }^{(13)}$. Elevated $\mathrm{HbA1c}$ levels are notably linked to severe coronary artery atherosclerosis ${ }^{(14)}$. The American Diabetes Association, in its recent position statement, stated that lowering $\mathrm{HbA} 1 \mathrm{c}$ may be associated with a reduction in the microvascular, neuropathic, and possibly macrovascular complications of DM ${ }^{(15)}$. Further, they provide evidence that the risk for developing CAD is significantly higher with elevated $\mathrm{HbA} 1 \mathrm{c}$ levels even within the normal range ${ }^{(\mathbf{1 6})}$.

The revascularization strategy is determined by evaluating the complexity of the overall lesions in coronary angiography. However, many studies demonstrated that the mis-estimation may occur in visual assessment of coronary angiography, which may result in inappropriate revascularization ${ }^{(\mathbf{1 7})}$. In Leape et al. (18) study, they found that imprecise reading of angiogram led to an overestimation of appropriate use of coronary artery bypass graft (CABG) by $17 \%$ and of percutaneous trans-luminal coronary angioplasty by $10 \%$.

Synergy between percutaneous coronary intervention with taxus and cardiac surgery (SYNTAX) score is a tool to comprehensively evaluate the overall severity of coronary lesions based on visual interpretation of angiogram in patients with at least one $\geq 50 \%$ coronary stenosis ${ }^{(\mathbf{1 9})}$.

In the present study, we analyzed the prevalence and pattern of coronary artery disease in $\mathbf{5 0}$ diabetic patients with cardiomyopathy who underwent elective coronary angiography for evaluation of suspected CAD. The evaluation of analysis was assessed by objective methods which were done by HbAlc, echocardiography and SYNTAX score to estimate the severity of CAD through coronary angiography.

Our present study results revealed that there was highly statistically significant ( $p$-value $<0.001)$ Positive correlation $(r=0.82)$ between $\mathrm{HbAlc}$ and duration of DM.

In our present study, the analysis of clinical data was as follow; as regard $\mathrm{HbA1c}$, the mean $\mathrm{HbA1c}$ of all studied patients was $7.4 \pm 1.03 \%$ with minimum $\mathrm{HbA} 1 \mathrm{c}$ of $5.5 \%$ and maximum HbA1c of $10 \%$.

As regard SYNTAX score in our study, the mean score of all studied patients was $14.2 \pm 5.6$ with range from $0-28$. As regard number of vessels, there were 16 patients $(32 \%)$ with single vessel CAD, 8 patients $(16 \%)$ with Two Vessels CAD / Significant LM CAD, 5 patients (10\%) with Three Vessels CAD, 20 patients (40\%) with Atherosclerotic non-significant Coronaries while there was 1 patient (2\%) with normal coronary angiography.

Regarding to our study results there was highly statistically significant (p-value $<0.001)$ positive correlation between duration of diabetes mellitus and SYNTAX score. Srinivasan et al. (20) study revealed a significant increase in the mean SYNTAX score, vessel score, and coronary collateral grade observed between 5 and 10 years of diabetic duration when compared to less than 5 years of diabetes, whereas the difference in the mean SYNTAX score, vessel score, and coronary collateral grade both in the nondiabetics and less than 5 years of diabetes, 5-10 years, and more than 10 years of diabetes was not significant. The observation from the Srinivasan et al. (20) study indicated that the coronary profile between the nondiabetic group and the less than 5 years of diabetes could be similar and a chronic severe structural narrowing of coronary artery takes place in 5-10 years of type 2 diabetes mellitus. Beyond 10 years, they visually noticed a plateau trend with no further morphological changes in the coronary arteries. Huang et al. ${ }^{(21)}$ study had showed that the incidence of microvascular outcomes is higher after 10 years of type 2 diabetes mellitus and kept on progressing, whereas the cardiovascular complications seem to be appearing before 10 years of diabetes. Mather $\boldsymbol{e t}$ al. (22) study revealed that type 2 diabetes mellitus with more than 5 years of duration have quantitative and qualitative, severe vascular disease compared to less than 5 years of diabetes. Their finding raised the interesting possibility of insulin resistance in generation of macrovascular complications in type 2 diabetes mellitus. Hyperinsulinemia brought about both functional and structural changes in the blood vessels.

Our study results revealed that there was highly statistically significant positive correlation between HbA1c and SYNTAX score. In contrast to our study, Ul-Haque et al. ${ }^{(23)}$ study revealed that; age $>53$ was found to be a predictor for SYNTAX score $\geq 23$ in diabetic patients $(\mathrm{p}<0.05)$. Male gender and smoking were found to be independent predictors for three-vessel disease in the non-diabetic population ( $p$ value $<0.05$ ). There was no significant relationship between the SYNTAX score and HbA1c levels in nondiabetics $(\mathrm{p}=0.885)$ and diabetics. Similar to our results, Sahal et al. ${ }^{(24)}$ study, screened 905 patients with $\mathrm{CAD}$, and their results strongly correlated disease severity and higher SYNTAX score $(\geq 23)$ with elevated $\mathrm{HbA} 1 \mathrm{c}$ levels in diabetics. In consistent to our study, Kapil et al. ${ }^{(25)}$ study, revealed a positive predictor relationship between $\mathrm{HbAlc}$ and cardiovascular disease $(\mathrm{p}=0.001)$. In consistent to our study, Dutta et al. (26) study revealed that the SYNTAX score also reported a highly significant correlation between elevated $\mathrm{HbA} 1 \mathrm{c}$ and higher SYNTAX score. Also, in consistent to our results, Basman et al. ${ }^{(27)}$ study, found a positive correlation of $\mathrm{HbA1c}$ with both the presence of CAD and the severity of CAD, as quantified by the SYNTAX score. We found that higher $\mathrm{HbA} 1 \mathrm{c}$ levels did not only predict the presence of CAD, but also the severity of CAD (as 
measured by the SYNTAX scores). HbA1c is known to be an independent predictor for the severity of CAD (27). While in Hong et al. ${ }^{(28)}$, Saleem et al. ${ }^{(29)}$, studies; had shown that in the patients with diabetes, HbA1c is an independent risk factor for CAD.

In non-diabetic patients, Hong et al. ${ }^{(28)}$, Liu et al. (30), Garg et al. ${ }^{(31)}$ studies, had suggested that high-normal glucose and HbA1c level in patients without diabetes are associated with a higher risk of CAD. Also, in Gustafsson et al. ${ }^{(32)}$, Cicek et al. ${ }^{(33)}$ studies had demonstrated that an elevated $\mathrm{HbA1c}$ level is associated with poor outcomes in patients presenting with acute coronary syndrome

In our prospective study, we have shown that severity of CAD in diabetic patients is strongly related to increase of $\mathrm{Hb} 1 \mathrm{AC}$ and also with the increase of diabetes mellitus duration.

Glycated hemoglobin has a strong effect in structure and the function of diabetic heart so we suggest that glycemic control in those with diabetes is a critical mechanism in the prevention of cardiac dysfunction and heart failure.

SYNTAX score commonly assesses coronary artery disease complexity by invasive coronary angiography so we suggest that SYNTAX score reduced inappropriate coronary revascularization in stable patients with $\mathrm{CAD}$ and it will help in evaluating and selecting the most appropriate revascularization strategy among diabetic cardiomyopathic patients. However, further controlled studies are needed to validate our findings in a larger cohort of patients.

\section{CONCLUSION}

There was a correlation between SYNTAX score and both of $\mathrm{HbAlc}$ and duration of diabetes.

\section{REFERENCES}

1. American Diabetes Association (2014): Diagnosis and classification of diabetes mellitus. Diabetes Care, 37:8189.

2. Seferovic Mitrovic J, Seferovic P, Vujisic Tesic B et al. (2012): Predictors of diabetic cardiomyopathy in asymptomatic patients with type 2 diabetes. Int $\mathbf{J}$ Cardiol., 156:219-221.

3. Boonman-De Winter L, Rutten F, Cramer M et al. (2012): High prevalence of previously unknown heart failure and left ventricular dysfunction in patients with type 2 diabetes. Diabetologia, 55: 2154-2162.

4. Fang Z, Yuda S, Anderson V et al. (2003): Echocardiographic detection of early diabetic myocardial disease. J Am Coll Cardiol., 41: 611-617.

5. Thomas C, Yong Q, Rosa $R$ et al. (2014): Cardiacspecific suppression of $\mathrm{NF}-\kappa \mathrm{B}$ signaling prevents diabetic cardiomyopathy via inhibition of the reninangiotensin system. Am J Physiol Heart Circ Physiol., 307: H1036-H1045.

6. Nakashima Y, Wight T, Sueishi K (2008): Early atherosclerosis in humans: role of diffuse intimal thickening and extracellular matrix proteoglycans. Cardiovasc Res., 79:14-23.
7. Beaglehole R, Magnus $\mathbf{P}$ (2002): The search for new risk factors for coronary heart disease: occupational therapy for epidemiologists? Int J Epidemiol., 31: 11171122 .

8. Solomon S, Bulwer B, Janardhanan $R$ (2007): Echocardiographic Assessment of ventricular systolic function. In Essential Echocardiography. Humana Press, Pp: 89-117.

9. Andova V, Georgievska-Ismail L, Srbinovska E et al. (2018): Does the Presence of Diabetes Mellitus Make a Difference in Pharmacological Stress Echocardiography Outcome Results? Open Access Maced J Med Sci., 6 (11): 2084-2090.

10. Mahadeva S, D'Souza SC, Padmanabha K (2014): Comparison of severity of coronary artery disease in diabetic and non-diabetic subjects using Gensini score in Indian subjects. J Diabetes Metab., 5:469-74.

11. National Kidney Foundation (2007): KDOQI clinical practice guidelines and clinical practice recommendations for diabetes and chronic kidney disease. Am J Kidney Dis., 49: 1-180.

12. Ul-Haque I, Deen Z, Shafique S et al. (2019): The Role of Glycated Hemoglobin A1c in Determining the Severity of Coronary Artery Disease in Diabetic and Non-Diabetic Subjects in Karachi. Cureus, 11(6): 498288.

13. Bastawesy R, Abdelmoniem A, Abdelkader $M$ et al. (2016): The relation between glycated hemoglobin and severity of coronary artery disease in non-diabetic patients with acute coronary syndrome. Int J Adv Res., 4: 2393-2399.

14. Cai A, Li G, Chen J et al. (2014): Glycated hemoglobin level is significantly associated with the severity of coronary artery disease in non-diabetic adults. Lipids Health Dis., 13: 181-85.

15. American Diabetes Association (2008): Nutrition recommendations and interventions for diabetes. Diabetes Care, 31:61-78.

16. Verdoia M, Schaffer A, Cassetti E et al. (2014): Glycosylated hemoglobin and coronary artery disease in patients without diabetes mellitus. Am J Prev Med., 47: 9-16.

17. Zhang H, Mu L, Hu S et al. (2018): Comparison of physician visual assessment with quantitative coronary angiography in assessment of stenosis severity in China. JAMA Inter Med., 178: 239-247.

18. Leape L, Park R, Bashore T et al. (2000): Effect of variability in the interpretation of coronary angiograms on the appropriateness of use of coronary revascularization procedures. Am Heart J., 139: 106113.

19. Sianos G, Morel M, Kappetein A et al. (2005): The SYNTAX score: an angiographic tool grading the complexity of coronary artery disease. EuroIntervention, 1: 219-227.

20. Srinivasan $M$, Kamath $P$, Bhat $N$ et al. (2016): Severity of coronary artery disease in type 2 diabetes mellitus: Does the timing matter? Indian Heart J., 68 (2): $158-163$.

21. Huang E, Laiteerapong N, Liu J et al. (2014): Rates of complications and mortality in older patients with diabetes mellitus: the diabetes and aging study. JAMA Intern Med., 174: 251-258. 
22. Mather K, Steinberg H, Baron A (2013): Insulin resistance in the vasculature. J Clin Invest., 123: 20132014.

23. Ul-Haque I, Deen Z, Shafique S et al. (2019): The Role of Glycated Hemoglobin A1c in Determining the Severity of Coronary Artery Disease in Diabetic and Non-Diabetic Subjects in Karachi. Cureus , 11 (6): 498286.

24. Sahal N, Farrag A, Ammar W et al. (2016): Impact of glycated hemoglobin level on severity of coronary artery disease in non-diabetic patients. J Cardiol Clin Res., 7: 258-63.

25. Kapil C, Rajasekhar D, Vanjakshamma V et al. (2018): Role of CIMT, eGFR, and serum HbA1c in predicting $\mathrm{CAD}$ in non-diabetic patients undergoing elective coronary angiography. World J Cardiovasc Dis., 8: $1-8$.

26. Dutta B, Neginhal M, Iqbal F (2016): Glycated hemoglobin (HbA1c) correlation with severity of coronary artery disease in non-diabetic patients-a hospital based study from North-Eastern India. J Clin Diagn Res., 10: 20-23.

27. Basman C, Fishman S, Avtanski D et al. (2020): Glycosylated hemoglobin, but not advanced glycation end products, predicts severity of coronary artery disease in patients with or without diabetes. Metabol Open, 7: 100050-6.
28. Hong L, Li X, Guo Y et al. (2014): Glycosylated hemoglobin A1c as a marker predicting the severity of coronary artery disease and early outcome in patients with stable angina. Lipids Health Dis., 13: 89-94.

29. Saleem T, Hameedullah K, Moataz M et al. (2008): Association of glycosylated haemoglobin level and diabetes mellitus duration with the severity of coronary artery disease. Diabetes Vasc Dis Res., 5:184-189.

30. Liu Y, Yang Y, Zhu J et al. (2011): Prognostic significance of hemoglobin Alc level in patients hospitalized with coronary artery disease. A systematic review and meta-analysis. Cardiovasc Diabetol., 10: 9894.

31. Garg N, Moorthy N, Kapoor A et al. (2014): Hemoglobin A1c in nondiabetic patients: an independent predictor of coronary artery disease and its severity. Mayo Clin Proc., 89 (7): 908-916.

32. Gustafsson I, Kistorp C, James M et al. (2007): Unrecognized glycometabolic disturbance as measured by hemoglobin A1c is associated with a poor outcome after acute myocardial infarction. Am Heart J., 154 (3): 470-476.

33. Cicek G, Uyarel H, Ergelen M et al. (2011): Hemoglobin A1c as a prognostic marker in patients undergoing primary angioplasty for acute myocardial infarction. Coron Artery Dis., 22 (3): 131-137. 